\title{
Attending icu physicians and residents do not agree about the applicability of advance directives
}

\author{
NH Leder*, D Schwarzkopf, K Reinhart, C Hartog \\ From ESICM LIVES 2015 \\ Berlin, Germany. 3-7 October 2015
}

\begin{abstract}
Introduction
In Germany, advance directives (AD) are written documents explaining what medical treatment the individual would prefer in the future, should that individual lack mental capacity. AD are legally binding. When a patient has lost mental capacity, the treating physician judges whether the $\mathrm{AD}$ is applicable or not. The $\mathrm{AD}$ is applicable if the patient's present condition matches the clinical scenario the patient specified in writing (in the following termed "applicability statement"). Common applicability statements are "in the event of a terminal illness" or "if I have irreversible organ failure". It is unknown how ICU physicians judge the applicability of a given ADs.
\end{abstract}

\section{Objectives}

To compare the judgment of attending, i.e. experienced intensivists and the judgment of residents in critical care training.

\section{Methods}

Prospective observational patient-based study in 4 multidisciplinary ICUs of a German university hospital. Patients were included if stayed $>48$ hours, were mentally incompetent and had a written AD. The structured interviews took place on the day of patient inclusion. Treating physicians were provided with verbatim copies of the relevant statements and asked whether these matched the medical situation. The study was IRB approved.

\section{Results}

Fifty ICU patients with ADs were included; 33 (66\%) patients were mechanically ventilated. $78 \%$ of AD contained pre-printed applicability statements. $84 \%$ of ADs contained 3 or more applicability statements. All ADs, had they been applicable, refused life-supporting measures.

University Hospital Jena, Jena, Germany
39 ICU physicians participated (14 consultants and 25 residents). Consultants had more experience with $\mathrm{AD}$ than residents $(p=0.003)$. Attendings judged overall 43 $\mathrm{AD}$, residents judged $46 \mathrm{AD}$. A direct comparison of the judgment of both groups was possible in 39 cases. Physicians judged that 17 of $39 \mathrm{AD}$ were applicable at the time of the interview, but attendings' and residents' judgment agreed in only 6 cases. Cohens Kappa regarding the agreement of judgments was 0.13 [95\% confidence intervall $-0.09,0.35]$, i.e. there was no significant agreement between the groups. AD contained overall 153 single applicability statements, of which 25 were judged as applicable, i.e. that the current clinical condition matched the patient's written statement. But only 5 statements were judged applicable by both groups (Cohens Kappa $0.17[0.03,0.31])$, indicating no significant agreement.

\section{Conclusions}

Experienced and inexperienced intensivists vary considerably in their interpretation of an AD in the ICU. Given that the number of patients with an AD is growing, residency training should include structured education on how to interpret an $\mathrm{AD}$ and respect the patient's preference even in the challenging setting of an ICU.

\section{Grant Acknowledgment}

The study was partly funded by the German Ministry for Education and Research and the Interdisciplinary Center for Clinical Research of the Jena University Hospital.

Published: 1 October 2015

doi:10.1186/2197-425X-3-S1-A36

Cite this article as: Leder et al:: Attending icu physicians and residents do not agree about the applicability of advance directives. Intensive Care Medicine Experimental 2015 3(Suppl 1):A36.

\section{SpringerOpen ${ }^{\circ}$}

(C) 2015 Leder et al.; This is an Open Access article distributed under the terms of the Creative Commons Attribution License (http:// creativecommons.org/licenses/by/4.0), which permits unrestricted use, distribution, and reproduction in any medium, provided the original work is properly cited. 